\title{
Necesidades educativas en la etapa infantil en menores con cardiopatías congénitas. Descripción de una cohorte y revisión de la bibliografía
}

\section{Educational needs of Infant Education children with congenital heart disease. Description of a cohort and a systematic review}

\author{
Ma Del Rosario Mendoza Carretero* \\ mamendoz@ucm.es \\ Susana Ares Segura** \\ susana.ares@salud.madrid.org \\ Belén Sáenz-Rico De Santiago*1 \\ bsaenzri@edu.ucm.es \\ * Universidad Complutense de Madrid, España \\ **Hospital Universitario La Paz de Madrid, España
}

\section{Resumen:}

Las cardiopatías congénitas (CC) constituyen una de las principales causas de mortalidad en España. Con este estudio se quiere identificar la existencia de (co) morbilidades en el neurodesarrollo de estos menores para identificar y evidenciar la necesidad de recibir una intervención educativa los más pronto posible a través de programas de Atención Temprana (AT) para optimizar su desarrollo madurativo y al mismo tiempo ofrecer una revisión sistemática a modo de estado de la cuestión de este campo de intervención. Se valoró, con la Escala de Desarrollo Infantil Bayley-III, a infantes cardiópatas $(M=$ 25.3 meses), en el Hospital Universitario

\begin{abstract}
:
Congenital Heart Disease (CHD) is one of the leading causes of mortality in Spain. This study aims (i) to identify the existence of (co)morbidities in the neurodevelopment of a sample of these children and (ii) to demonstrate the need for them to receive educational intervention as soon as possible through early care programs so that their maturational development can be optimized. This study also presents a systematic review of the research available in the field. With the Bayley-III Child Development Scale, cardiac infants $(M=$ 25.3 months) at the University Hospital La Paz, at the Hospital 12 de Octubre and the Menudos Corazones Foundation were assessed. The biomedical and developmen-
\end{abstract}

1 Dirección para correspondencia (correspondence address):

Belén Sáenz-Rico de Santiago. Facultad de Educación. Dpto. Estudios Educativos. c/ Rector Royo Villanova, 1, 28040, Madrid (España) 
Necesidades educativas en la etapa infantil en menores con cardiopatías congénitas. Descripción de una cohorte y revisión de la bibliografía

Ma Del Rosario Mendoza Carretero, Susana Ares Segura y Belén Sáenz-Rico De Santiago

La Paz, en el Hospital 12 de Octubre y en la Fundación Menudos Corazones. Se describen las características biomédicas y de desarrollo de 90 participantes. El 68\% presenta un desarrollo cognitivo dentro del promedio, sucediendo lo mismo en el área del lenguaje $(42 \%)$ y motor $(44 \%)$. En estas últimas se evidencia un desarrollo normal-lento (35\% y $24 \%$, respectivamente) y limítrofe $(15 \%$ y $12 \%)$. La revisión realizada de la literatura científica al respecto corrobora que tanto el área comunicativa-lingüística como la motora son las dimensiones del desarrollo que presentan necesidades.

\section{Palabras clave:}

Necesidades educativas; cardiopatía congénita; desarrollo infantil; prevención; atención temprana. tal characteristics of 90 participants are described. $68 \%$ have an average cognitive development. Average rates were also reported for linguistic (42\%) and motor (44\%) development. For the latter category, normal-slow (35\% and $24 \%$, respectively) and bordeline (15\% and 12\%) development rates were found. The review of the scientific literature in this regard corroborates that the communicative-linguistic and the motor areas are the poorest dimensions of development.

\section{Key words:}

Educational needs; congenital heart disease; child development; prevention; early care.

\section{Résumé :}

Les cardiopathies congénitales (CHD) sont l'une des principales causes de mortalité en Espagne. Cette étude vise à identifier l'existence de (co)morbidités dans le développement neurologique de ces enfants afin d'identifier et de démontrer la nécessité de recevoir une intervention éducative le plus tôt possible à travers des programmes d'intervention précoce (IPE) pour optimiser leur développement maturatif et en même temps d'offrir une revue systématique comme état de l'art dans ce domaine d'intervention. Les nourrissons cardiopathes ( $M=25,3$ mois) ont été évalués à l'aide de l'échelle de développement de I'enfant Bayley-III à I'Hospital Universitario La Paz, I'Hospital 12 de Octubre et la Fundación Menudos Corazones. Les caractéristiques biomédicales et de développement de 90 participants sont décrites. Le développement cognitif se situait dans la moyenne dans 68 $\%$ des cas, et il en allait de même pour le langage (42\%) et les capacités motrices (44\%). Chez ces derniers, on constate un développement normal-lent (35\% et $24 \%$, respectivement) et limite (15\% et $12 \%)$. La revue de la littérature scientifique effectuée à cet égard corrobore que les domaines communicatif-linguistique et moteur sont les dimensions du développement qui présentent des besoins.

\section{Mots clés:}

Besoins éducatifs; cardiopathie congénitale; développement de l'enfant; prévention; attention précoce

Fecha de recepción: 16-01-2020

Fecha de aceptación: 22-06-2020 
Necesidades educativas en la etapa infantil en menores con cardiopatías congénitas. Descripción de una cohorte y revisión de la bibliografía

Ma Del Rosario Mendoza Carretero, Susana Ares Segura y Belén Sáenz-Rico De Santiago

\section{Introducción}

La educación es la base del desarrollo personal; se inicia a temprana edad, tras el nacimiento del infante, en la interacción con el entorno que le rodea, y se prolonga durante toda su vida. Los primeros años de vida de un niño o de una niña son fundamentales para alcanzar un óptimo desarrollo en edades posteriores. La estimulación temprana, entre otras opciones de intervención, a través de la comunicación y el juego, las vivencias relacionales con el contexto, repercute directamente en las conexiones neuronales del infante, y por ende en su neurodesarrollo. Los menores con cardiopatías congénitas, sometidos a intervención quirúrgica mediante cirugía por circulación extracorpórea² (CEC), en la actualidad al no considerarse sujetos de alto riesgo no pueden acceder a este tipo de servicios, lo cual puede afectar negativamente a su desarrollo.

El principal objetivo del estudio que se presenta a continuación es identificar las necesidades educativas mediante el estudio del neurodesarrollo de los menores con cardiopatías congénitas para evidenciar, desde una actitud preventiva, la necesidad de que se incorporen a programas de Atención Temprana (AT) tras su nacimiento e intervención quirúrgica, con la finalidad de reducir las morbilidades y comorbilidades que pueden evidenciarse en edades posteriores y, a su vez, realizar una revisión de la bibliografía para comparar los datos obtenidos en este estudio con los resultados de las investigaciones publicadas hasta la fecha.

\section{Marco teórico}

Las cardiopatías congénitas (CC), también denominadas malformaciones de los grandes vasos sanguíneos, son definidas como un defecto en la estructura del corazón presente en el feto y/o en el recién nacido (SECPCC, 2014), pues pueden ser detectadas durante el embarazo, en el nacimiento o en edades posteriores (OMS, 2020). Estas anomalías, con una tasa de incidencia de 8 por cada 1.000 nacidos vivos (SalamancaZarzuela, Morales-Luego, Alcalde-Martín, \& Centeno-Malfaz, 2018),

2 Cirugía por circulación extracorpórea: técnica que asume la función del corazón y de los pulmones durante la cirugía mediante un circuito oxigenador y una bomba que permite al cirujano trabajar en el corazón que no late, mientras que el cerebro continua funcionando (Greco, 2016). 
son consideradas una de las principales causas de mortalidad infantil en España (Pérez-Lescure, Mosquera, Latasa, \& Crespo, 2017). En ellas, predomina ligeramente el género masculino, especialmente en aquellas cardiopatías con obstrucción en la zona de salida de la sangre del ventrículo izquierdo, aunque el ductus y la comunicación interauricular son más frecuentes en el sexo femenino (García, 2006, pp.71).

La etiología de esta enfermedad crónica se desconoce con exactitud, aunque algunos autores como Villagrá (2019) indican que es probable que las alteraciones genéticas puedan estar vinculadas, pero en la mayoría no está identificada. Pese a esto, existen algunos factores de riesgo como la edad de los padres (menores de 18 o mayores de 35 años), antecedentes familiares con cardiopatías congénitas, menores con síndromes asociados (síndrome de Down, de Edwards), factores maternos como lupus, alcoholismo, rubéola y fenilcetonuria, o adicción a drogas, que favorecen tener un hijo con cardiopatía (Villagrá, 2019).

A pesar de que las causas carecen de una definición plena, sí que se conocen las distintas anomalías cardiacas que pueden presentar los menores, las cuales pueden estar combinadas unas con otras (Brotons, 2019). La misma autora señala que las cardiopatías congénitas pueden clasificarse según su anatomía, siendo esta la más utilizada por los cardiólogos y, según su fisiopatología, dividiéndose en cianógenas o acianógenas (véase Tabla 1), esto es, con o sin disminución de la saturación de oxígeno en la sangre (Fundación Salud Infantil, 2019).

Tabla 1

Tipos de cardiopatías congénitas en función de su fisiopatología

\begin{tabular}{|c|c|c|}
\hline \multirow{3}{*}{ 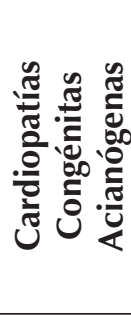 } & $\begin{array}{l}\text { Cortocircuito } \\
\text { de izquierda a } \\
\text { derecha }\end{array}$ & $\begin{array}{l}\text { Comunicación Interventricular, Comunicación } \\
\text { interauricular, ductus, canal aurículo-ventricular, } \\
\text { drenaje venoso anómalo pulmonar parcial. }\end{array}$ \\
\hline & $\begin{array}{l}\text { Obstructivas co- } \\
\text { razón izquierdo }\end{array}$ & $\begin{array}{l}\text { Coartación aórtica, estenosis aórtica, estenosis } \\
\text { mitral, hipoplasia del ventrículo izquierdo. }\end{array}$ \\
\hline & $\begin{array}{l}\text { Insuficiencias } \\
\text { valvulares y otras }\end{array}$ & $\begin{array}{l}\text { Insuficiencia mitral, insuficiencia aórtica, esteno- } \\
\text { sis ramas pulmonares. }\end{array}$ \\
\hline \multirow{3}{*}{ 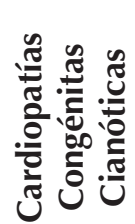 } & $\begin{array}{l}\text { Obstructivas co- } \\
\text { razón derecho }\end{array}$ & $\begin{array}{l}\text { Tetralogía de Fallot, atresia pulmonar, ventrículo } \\
\text { único. }\end{array}$ \\
\hline & Mezcla total & $\begin{array}{l}\text { Ventrículo único, truncus arterioso, drenaje } \\
\text { venoso anómalo pulmonar total. }\end{array}$ \\
\hline & Falta de mezcla & Transposición de las Grandes Arterias \\
\hline
\end{tabular}

Fuente: Fundación Salud Infantil, 2019. 
Gracias a los avances producidos en la cardiología de diagnóstico, en los procedimientos, en las técnicas y en los cuidados perioperatorios (Williams, Fifer \& Andrews, 2015), en las últimas décadas, se ha reducido el índice de mortalidad en aquellos recién nacidos que presentan esta enfermedad (SECPCC, 2014), alcanzándose una tasa de supervivencia del 85\% aproximadamente (Martín, 2006, pp.118). Esta tasa de supervivencia permite reconocer lentificaciones en el neurodesarrollo que producen morbilidades y comorbilidades (Martínez-Biarge Jowett, Cowan \& Wusthoff, 2013) en edades posteriores, especialmente en niños y niñas que han sido sometidos a cirugía por circulación extracorpórea y que se encuentran en edad escolar pues su desarrollo motor, cognitivo y habilidades atencionales son inferiores a lo esperado en comparación a su grupo de edad (Granberg, Rydberg \& Fisher, 2008).

En España, la educación se corresponde con etapas no obligatorias, como la educación infantil (0-6), estructurada en dos ciclos de tres años cada una, y con obligatorias como la Ed. Primaria (6-11) y la Educación Secundaria Obligatoria (12-16), contribuyendo al desarrollo y a la formación del ser humano. Sin embargo, este estudio está centrado en la primera infancia, concretamente de 0 a 42 meses; de ahí la importancia de la primera etapa del sistema educativo, la educación infantil, pues esta favorece el desarrollo físico, sensorial, intelectual, afectivo y social de los infantes; así como de la importancia de participar en programas de AT en caso de necesidad.

Algunos infantes con CC sometidos a CEC, por recomendación médica en las revisiones periódicas, deben evitar la incorporación a escuelas infantiles hasta los dos años aproximadamente, o hasta el inicio del segundo ciclo de esta etapa, a fin de reducir el contagio por infecciones. Esta falta de participación en el sistema educativo puede generar lentificaciones en su desarrollo pues, generalmente, el infante queda al cuidado de algún miembro de la familia, y debido al suceso traumático experimentado, este tiene tendencia a sobreprotegerle.

En ocasiones, los menores con esta patología participan en programas de AT, tras una valoración previa y la emisión de un informe, a fin de detectar, prevenir riesgos en su desarrollo y/o paliar las necesidades presentes en el mismo. Según el Decreto 46/2015, de 7 de mayo, la AT se entiende como un conjunto de intervenciones de carácter sanitario, educativo y de servicios sociales que están dirigidas a la población infantil, a su familia y a su entorno, para dar respuesta a las necesidades, 
transitorias o permanentes, que puedan presentan los infantes con trastornos en su desarrollo o con riesgo de padecerlos (BOCM, 2015).

Pese a ello, esta población, en muchas comunidades autónomas, carece de reconocimiento como infantes de riesgo biológico, tal y como sucede en la Comunidad de Madrid pues no está contemplada en los Criterios de Ayuda a la Derivación, preferentes y ordinarios, a Recursos de Atención Temprana (Protocolo de Coordinación de Atención Temprana, 2018). Por consiguiente, para que un niño o una niña con cardiopatías congénitas puedan participar en programas de AT, es necesario que los especialistas médicos como los neonatólogos, cardiólogos pediátricos, etc., le deriven a este servicio. Sin embargo, a nivel nacional, algunas comunidades autónomas como Galicia sí les incluye en el Protocolo de Coordinación, Intervención y Derivación Interinstitucional en Atención Temprana, concretamente en el eje I., denominado factores de riesgo biológico y, por consiguiente, pueden incorporarse en estos programas.

La ausencia de participación de estos menores en los dos ámbitos fundamentales de estimulación en la primera infancia, como son la Educación Infantil y la AT, está repercutiendo negativamente en su desarrollo, pudiendo conducir a morbilidades y comorbilidades en edades académicas posteriores.

\section{Morbilidades y comorbilidades halladas en los menores con cardiópatías}

Según la literatura científica, las lentificaciones en el neurodesarrollo de los menores con CC son evidentes y palpables. Ya 1979, Azcoaga defendía que el tipo de intervención quirúrgica mediante CEC producía lentitud en las funciones cerebrales superiores y que a mayor duración de la misma, podía existir mayor riesgo de deterioro (Hövel-Gürich et al., 2007; Sananes et al., 2012; McCusker, Armstrong, Mullen, Doherty, \& Casey, 2013).

En base a esto, es preciso destacar que se manifiestan morbilidades en el área cognitiva, en el área comunicativo-lingüística y en la motora. Algunos estudios científicos muestran que existen deficiencias en las funciones ejecutivas, como por ejemplo en las habilidades de planificación y organización, memoria de trabajo, autorregulación, etc. (Calderon et al., 2012; Bellinger et al., 2011), y estas disfunciones pueden afectar negativamente a muchos aspectos del desarrollo de los niños, asociándose directamente 
con la preparación escolar y con un menor rendimiento académico, pues presentan dificultades en la resolución abstracta de problemas, en el razonamiento inferencial (Bellinger et al., 2003), y en la lecto-escritura (Blair \& Razza, 2007), aunque el Cociente Intelectual (Cl) se mantiene dentro del promedio general de la población (Calderon et al., 2010).

Algunos autores coinciden en que los déficits de las funciones ejecutivas persisten hasta la adolescencia, acompañándose de inflexibilidad cognitiva (Bellinger, et al., 2010; Cassidy, White, DeMaso, Newburguer, \& Bellinger, 2014), aunque otro análisis reveló que las habilidades básicas o de menor nivel, como la secuencia alfabética, estaban intactas. Sin embargo, los jóvenes adultos con cardiopatía presentaron dificultades para coordinar las destrezas iniciales, como extraer y comprender el significado de un discurso o aplicar conceptos matemáticos para resolver un problema, mostrando un desarrollo inferior en la memoria de trabajo y en las capacidades organizativas (Bellinger et al., 2003). Estos hallazgos muestran la necesidad de utilizar estrategias para prevenir o limitar estos rasgos de aprendizaje y, además, es necesario realizar un seguimiento, ya que muchos de ellos se muestran en edades escolares y no en la primera infancia (Creighton et al., 2007).

Las lentificaciones en el lenguaje de los menores con CC son evidentes en el primer examen del neurodesarrollo encontrado en la literatura (Hövels-Gürich \& McCusker, 2016) y aparecen cuando presentan un nivel inferior a su edad debido a la inmadurez (Whitehurst \& Fischel, 1994), lo cual genera dificultades en las estructuras, en el significado y en el uso social de la lengua (Bishop, 2006), aunque el subárea del lenguaje receptivo y el razonamiento verbal estaban dentro del promedio poblacional (McCusker et al., 2013). Siguiendo a Monfort y Juárez (2010), el lenguaje se va a ver influenciado por el ambiente, esto es, que si no existen estímulos exteriores o éstos son escasos puede disminuir la cantidad y la calidad del mismo. La falta de información apropiada puede estar relacionada con el gran número de madres y padres que limitan las actividades del menor con cardiopatía (LeRoy et al., 2003).

Autores como Sananes at al. (2012) indican que los retrasos motores tempranos pueden deberse a las restricciones asociadas en la exploración y a la estancia hospitalaria pues durante la misma carecen de estímulos. Los retrasos en sus subáreas, fina y gruesa, se identificaron a una edad temprana (12 meses), alcanzándose un desarrollo inferior doce meses después. 
A pesar de esto, se observa una recuperación a lo largo del tiempo en los sustratos neurológicos del funcionamiento motor ya sea debido a la plasticidad cerebral o porque los menores entrenen estas dificultades (Hövels-Gürich \& McCusker, 2016). Sin embargo, estos déficits parecen ser preponderantes $y$, aunque se produce una aparente recuperación, emergen dificultades en otros dominios como por ejemplo en procesos intelectuales superiores (Karsdorp, Everaerd, Kindt, \& Mulder, 2007; Snookes et al., 2010).

En mayor medida, las tres áreas muestran alteraciones y eso repercute en el desarrollo del menor con CC, las cuales se hacen más evidentes en edades posteriores, apareciendo morbilidades y comorbilidades en los niveles académicos superiores.

\section{Marco empírico}

Este estudio, de carácter cuantitativo, transversal y multicéntrico, se realizó a través de la técnica de muestreo no probabilístico por conveniencia. Los participantes fueron seleccionados en base a los siguientes criterios de inclusión: (1) debían presentan cardiopatías congénitas, (2) sin anomalías cromosómicas asociadas y/o síndromes genéticos, (3) nacimientos a término, (4) haber sido intervenidos quirúrgicamente mediante CEC antes del primer año de vida y (5) no superar los 42 meses de edad.

Con relación a lo anterior, cabe destacar que la cohorte está formada por un total de 90 menores diagnosticados de CC, sin anomalías cromosómicas ni síndromes genéticos asociados, intervenidos quirúrgicamente mediante la técnica de circulación extracorpórea antes del primer año de vida, con una duración media de 163 min., (rango: 27-960 minutos). La mayoría de la muestra presenta Transposición de las Grandes Arterias (TGA) (29\%) y Síndrome del Corazón Izquierdo Hipoplásico (HLHS) (15\%) (Véase Tabla 2). La edad media resultó de 25.3 meses, con predominio masculino (68\%). Se recogió una edad gestacional media de 37.3 semanas (rango: 37 - 41 semanas). El 98\% fue parto único y presentaron una puntuación de Apgar media de 8, al minuto de nacer, y 9, a los cinco minutos. La somatometría en el nacimiento fue la siguiente: peso medio, 2.975 g (rango: $2030 \mathrm{~g}-4800 \mathrm{~g}$ ), longitud media, $46.7 \mathrm{~cm}$ (rango: $44 \mathrm{~cm}-53 \mathrm{~cm}$ ) y perímetro craneal medio, $33 \mathrm{~cm}$ (rango: $31 \mathrm{~cm}-36.5$ ). 
Necesidades educativas en la etapa infantil en menores con cardiopatías congénitas. Descripción de una cohorte y revisión de la bibliografía

Ma Del Rosario Mendoza Carretero, Susana Ares Segura y Belén Sáenz-Rico De Santiago

Tabla 2

Tipología de los menores con cardiopatías congénitas

\begin{tabular}{|c|c|c|c|}
\hline Tipología & $\mathrm{N}(\%)$ & Diagnóstico & $\mathrm{N}(\%)$ \\
\hline $\begin{array}{c}\text { Transposición de las grandes } \\
\text { arterias (TGA) }\end{array}$ & $19(24 \%)$ & $\begin{array}{c}\text { Síndrome izquierdo hipoplá- } \\
\text { sico }\end{array}$ & $12(15 \%)$ \\
\hline Tetralogía de Fallot & $7(9 \%)$ & Canal Aurículo-Ventricular & $6(8 \%)$ \\
\hline Coartación de Aorta & $6(8 \%)$ & $\begin{array}{l}\text { Comunicación interventricu- } \\
\qquad \operatorname{lar}(\mathrm{CIV})\end{array}$ & $5(6 \%)$ \\
\hline $\begin{array}{l}\text { Comunicación Interauricular } \\
\qquad(\mathrm{ClA})\end{array}$ & $5(6 \%)$ & Estenosis aórtica & $3(4 \%)$ \\
\hline Cortriatriatum & $3(4 \%)$ & $\begin{array}{c}\text { Transposición de las Grandes } \\
\text { Arterias Corregida }\end{array}$ & $2(3 \%)$ \\
\hline Drenaje Venoso Anómalo & $1(1 \%)$ & $\begin{array}{l}\text { Ventriculo Derecho de Doble } \\
\text { Salida }\end{array}$ & $1(1 \%)$ \\
\hline $\begin{array}{l}\text { Complejo de Cavidades Iz- } \\
\text { quierdas Hipoplásicas }(\mathrm{CCIH})\end{array}$ & $1(1 \%)$ & $\begin{array}{c}\text { Insuficiencia tricúspidea } \\
\text { severa (ITS) }\end{array}$ & $1(1 \%)$ \\
\hline $\begin{array}{c}\text { TGA+CIV } \\
\text { Atresia pulmonar + CIV }\end{array}$ & $\begin{array}{l}3(4 \%) \\
1(1 \%)\end{array}$ & $\mathrm{TGA}+\mathrm{CIA}$ & $1(1 \%)$ \\
\hline
\end{tabular}

El desarrollo de los infantes se valoró a través de la Escala de Desarrollo Infantil Bayley-III, la cual permite una valoración del desarrollo en tres dominios: cognitivo, motor (fino y grueso) y comunicativo-lingüístico (receptivo y expresivo), en un rango de edad de 0 meses- 15 días y 42 meses y 15 días. La primera escala evalúa el desarrollo sensoriomotor, la exploración, la manipulación, la relación entre objetos, la formación de conceptos, la memoria, entre otros aspectos del procesamiento cognitivo; la escala motora mide las habilidades manipulativas, asociadas con la motricidad fina, tiene en cuenta la prensión, la integración perceptivomotora, la planificación y la velocidad, mientras que la prueba de motricidad gruesa se encarga de valorar el movimiento en las extremidades superiores e inferiores, y el torso; la última escala, a través de la prueba de comunicación receptiva permite evaluar las conductas preverbales, el desarrollo del vocabulario y la comprensión de marcadores morfológicos, mientras que la expresiva, mide los balbuceos, las gesticulaciones, la referencia conjunta y los turnos de palabra, entre otros (Bayley, 2015).

Para realizar estas valoraciones, se contactó con los familiares de los menores con cardiopatías congénitas de dos formas distintas. Por un lado, la Fundación Menudos Corazones de Madrid envió a todos los socios familiares, que cumplían con los criterios de inclusión, una carta electrónica invitándoles a participar en el estudio. En ésta se explicaba 
en qué consistía y la finalidad del mismo. Una vez confirmada la participación, se realizaron Ilamadas telefónicas para detallar la prueba que se iba a utilizar en la valoración, así como su duración y el reporte a obtener tras la misma. Por otro lado, tanto el Hospital Universitario La Paz de Madrid como el Hospital Universitario 12 de Octubre contaron con el aval de los comités éticos para el desarrollo del estudio. Tras esto, se procedió a la búsqueda de participantes en las bases de datos hospitalarias, incluyendo los criterios de inclusión, facilitándole los datos de los infantes al investigador principal, el cual seguía el procedimiento mencionado con anterioridad.

Todas las valoraciones del neurodesarrollo de los menores con cardiopatías congénitas contaron con el consentimiento informado de los familiares y/o tutores legales de los menores. Tras la valoración, se realizaba un informe psicopedagógico por cada infante, que era enviado tanto a los progenitores como a los doctores que se encargaban de las revisiones y seguimiento del paciente. Cada uno de estos informes, recogía los resultados alcanzados a través de la Escala de Desarrollo Infantil Bayley-III y, en caso de necesidad, se pautaban las orientaciones para fomentar el desarrollo de las distintas áreas y la pertinente derivación al servicio de primera infancia correspondiente.

Las variables de estudio se codificaron en dos dimensiones: biomédicas y de neurodesarrollo. Las primeras se corresponden con datos antropométricos como la talla, el peso, el perímetro cefálico y, a su vez, se recopilaron datos sobre la edad gestacional, el test de Apgar, tipo de embarazo (único/múltiple), tipo de cardiopatía congénita y la duración de la intervención quirúrgica mediante CEC. Las segundas se componen de los resultados alcanzados en las distintas áreas del desarrollo (cognitiva, lenguaje, motora), las cuales también fueron analizadas de manera descriptiva a través de Microsoft Excel y, además, para comprobar si existía relación entre el tipo de cardiopatía (véase Tabla 2) y el desarrollo de las distintas áreas se utilizó la técnica estadística ANOVA Unifactorial y Correlación de Pearson a través del Software Statistical Package for the Social Sciences (SPSS). 


\section{Resultados y Discusión}

Se describen las características de 90 participantes con CC para conocer el neurodesarrollo de los menores con malformaciones en los grandes vasos sanguíneos. Esta investigación destaca por presentar una muestra representativa de la población de interés en España, que permite extrapolar los resultados obtenidos. La cohorte está constituida por niños en el $75 \%$, próximo a lo señalado en la literatura científica (Chen et al., 2014; Medoff-Cooper et al., 2016). La edad media de los sujetos de este estudio se sitúa en torno a los dos años de edad ( $M=25.3$ meses), asemejándose al resto de investigaciones.

Todos los participantes del estudio fueron valorados a través de la Escala de Desarrollo Infantil Bayley-III, desde el 2016 hasta el 2019, y obtuvieron las siguientes puntuaciones medias en las áreas del neurodesarrollo: cognitiva, 99.3 (rango: 60-140), comunicativa-lingüística, 87.3 (rango: 47-118) y motora, 91.3 (rango: 46-127). En esta última, a través de las puntuaciones escalares medias obtenidas a través de la escala de desarrollo, se identifica un mayor desarrollo en la subárea gruesa, 32.4 (rango: 5-65) en comparación con la fina, 24.5 (rango: 7-59) mientras que en el área del lenguaje se observa un desarrollo similar tanto en el lenguaje expresivo, 14.7 (rango: 4-43) como receptivo, 15.12 (rango: 6-35) en los participantes del estudio.

En base a esto, es importante mencionar que el $65 \%$ de los participantes de este estudio presentan un desarrollo cognitivo normal dentro del promedio (90-109), sucediendo lo mismo en el área del lenguaje (42\%) y en la motora (44\%). A pesar de esto, se evidencia que algunos sujetos presentan un desarrollo normal-lento (80-90) en estas dos últimas áreas ( $17 \%$ y el $24 \%$, respectivamente). A esto se le debe añadir, la existencia de participantes en un rango limítrofe (70-79) tanto en el lenguaje (15\%) como en el desarrollo motor (12\%).

Por ello, y a fin de determinar si existe o no correlación entre el tipo de cardiopatía y el desarrollo por áreas (véase Figura 1), se utilizó la técnica estadística ANOVA Unifactorial a través del Software SPSS. Para ello fue necesario agrupar los tipos de CC, mencionados con anterioridad, en 5 grupos (véase Tabla 3). 
Necesidades educativas en la etapa infantil en menores con cardiopatías congénitas. Descripción de una cohorte y revisión de la bibliografía

Ma Del Rosario Mendoza Carretero, Susana Ares Segura y Belén Sáenz-Rico De Santiago

Tabla 3

Agrupación de los tipos de CC para análisis de datos ANOVA Unifactorial

\begin{tabular}{clc}
\hline Grupo & Tipo de CC & Sujetos \\
\hline 1 & TGA & 33 \\
2 & CoA & 11 \\
3 & CAV & 23 \\
4 & Alteraciones valvulares & 14 \\
5 & Anomalías de tabiques (CIV, CIA...) & 9 \\
Total & & 90 \\
\hline
\end{tabular}

TGA: Transposición de las Grandes Arterias; CoA: Coartación de Aorta; CAV: Canal Aurículo-Ventricular; CIV: Comunicación Interventricular; CIA: Comunicación Interauricular.

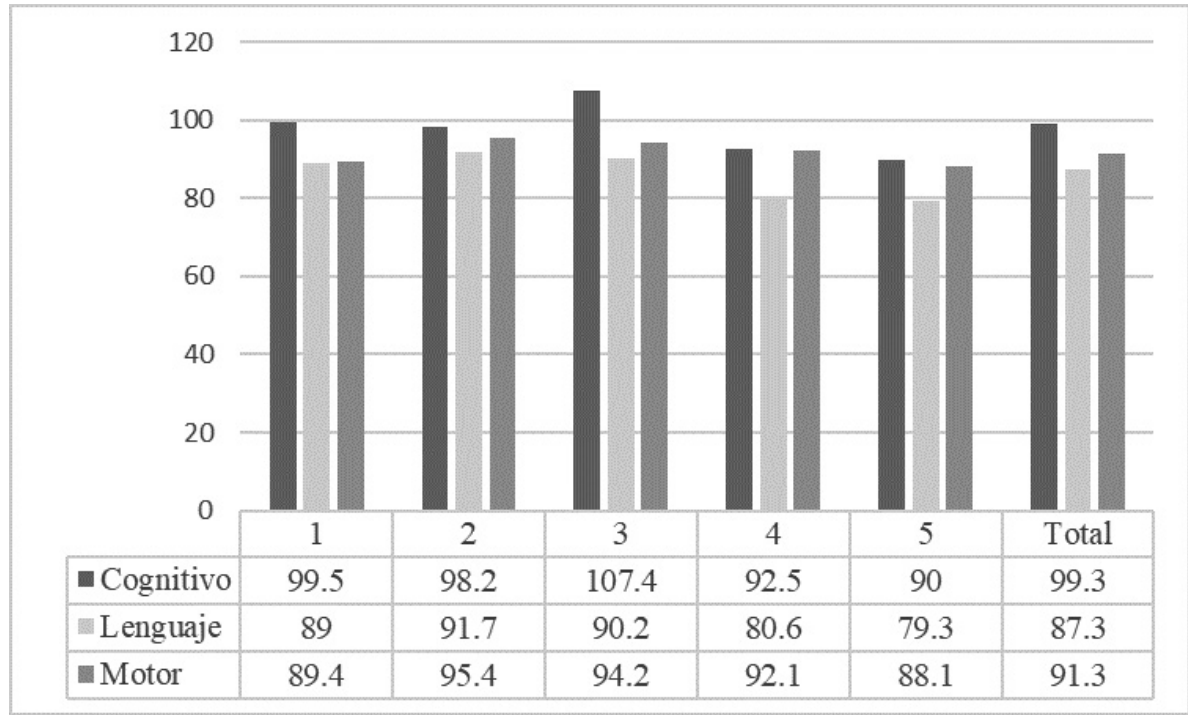

Figura 1. Gráfico descriptivo de relación entre el tipo de cardiopatía y el desarrollo por áreas

Los resultados de los cinco grupos analizados muestran que a nivel cognitivo, el grupo 3 tiene valores significativamente mayores que los demás $(M=107.4 ; D T=10.9)$ mientras que el grupo 5 obtiene puntuaciones inferiores $(M=90 ; D T=17.8)$ aunque se sitúa dentro del promedio poblacional (90-109); no obstante, es necesario destacar que este último grupo presenta una muestra inferior en comparación con el resto de grupos. En el área comunicativo-lingüística los grupos $2(M=$ $91.7 ; D T=7.9)$ y $3(M=90.2 ; D T=9.4)$ tienen mayores valores que el 
resto, especialmente frente a los grupos $4(M=80.6 ; D T=9.5)$ y $5(M=$ 87.3; $D T=12.2)$. En el desarrollo motor se observa como el grupo $1(\mathrm{M}$ $=88.4 ; D T=14.8)$ y $5(M=88.1 ; D T=18.8)$ obtienen menores valores que el resto de grupos mientras que el grupo 2 es el que obtiene una mayor puntación.

Es importante detallar que, de los cinco grupos analizados, tres obtienen puntuaciones por debajo del promedio en el área comunicativolingüística y dos a nivel motor; estos dos últimos coinciden con dos de los tres que presentan alteraciones en el lenguaje. Por ende, se puede determinar que los infantes con cardiopatías congénitas, valorados hasta los tres años y medio, pueden presentar lentificaciones en determinadas áreas del desarrollo y estas pueden ser detectadas tempranamente para realizar una intervención adecuada a las necesidades, transitorias y/o permanentes, que presenten los infantes.

Tras esto, se quiso conocer si existía relación entre las áreas del neurodesarrollo y los tipos de cardiopatías (véase Tabla 4), para lo cual se realizó la correlación de Pearson. Los resultados hallados muestran que existe una correlación significativa al nivel .05 entre el tipo de cardiopatía y el área del lenguaje $(p=.011<a=.05)$; sin suceder lo mismo en el resto de las áreas. No obstante, se pudo comprobar que existe una relación directa entre ellas.

Tabla 4

Correlaciones entre las áreas del neurodesarrollo y los tipos de cardiopatías

\begin{tabular}{llrrrr}
\hline & Cognitivo & Lenguaje & Motor & Tipo CC \\
\hline Cognitivo & Correlación de Pearson & 1 & $.692^{* *}$ & $.569^{* *}$ & -.182 \\
& Sig. (bilateral) & & .000 & .000 & .090 \\
& $\mathrm{~N}$ & 90 & 90 & 90 & 88 \\
\hline Lenguaje & Correlación de Pearson & $.692^{* *}$ & 1 & $.537^{* *}$ & $-.269^{*}$ \\
& Sig. (bilateral) & .000 & & .000 & .011 \\
& $\mathrm{~N}$ & 90 & 90 & 90 & 88 \\
\hline Motor & Correlación de Pearson & $.569^{* *}$ & $.537^{* *}$ & 1 & .050 \\
& Sig. (bilateral) & .000 & .000 & & .642 \\
& $\mathrm{~N}$ & 90 & 90 & 90 & 88 \\
\hline Tipo CC & Correlación de Pearson & -.182 & $-.269^{*}$ & .050 & 1 \\
& Sig. (bilateral) & .090 & .011 & .642 & \\
& $\mathrm{~N}$ & 88 & 88 & 88 & 88 \\
\hline
\end{tabular}

**La correlación es significativa en el nivel .01 (bilateral).

*La correlación es significativa en el nivel .05 (bilateral). 
Este estudio, cuya finalidad era identificar la existencia de alteraciones en el neurodesarrollo de menores con cardiopatías congénitas a fin de detectar las posibles necesidades educativas para su incorporación, o durante la permanencia, en el sistema educativo a la etapa infantil, realizado en la Comunidad Autónoma de Madrid (España), muestra que estos menores presentan lentificaciones en algunas áreas de su desarrollo.

Asimismo, y en continuación con el propósito del objetivo, los resultados alcanzados en esta cohorte se quisieron contrastar, a través de una revisión sistemática, con publicaciones presentes en la literatura científica, cuyas muestras cumplieran con los criterios de inclusión indicados con anterioridad (véase Figura 2). Para lograr tal fin, se realizó una revisión sistemática, correspondiente a la última década (2008-2018), consultado las bases de datos de Scopus y PubMed; se identificaron 143 investigaciones publicadas, se analizaron en profundidad, 117 , y se eliminaron, 102.

Posteriormente, se analizaron minuciosamente los 15 estudios restantes y se suprimieron todos aquellos que no habían utilizado como instrumento de evaluación la Escala de Desarrollo Infantil-Bayley, y aquellos que usaron más de una escala de medida con sus participantes, a fin de que los estudios presentados fueran similares. Por consiguiente, únicamente se pudieron incluir 4 investigaciones (véase Tabla 5). En base a esto, se presenta un análisis comparativo entre los hitos de neurodesarrollo en esta casuística frente a otros estudios publicados en la literatura científica actual.

Tabla 5

Neurodesarrollo en menores con cardiopatías congénitas, sin anomalías cromosómicas asociadas (comparativa de tres muestras)

Chen et al. Hallioglu et Medoff-Cooper Este estudio $(\mathrm{N}=24) \quad$ al. $(\mathrm{N}=61) \quad$ et al. $(\mathrm{N}=72) \quad(\mathrm{N}=90)$

\begin{tabular}{lllll}
\hline País & Estados Unidos Turquía & \multicolumn{2}{l}{ Estados Unidos } & España \\
\hline Año & 2015 & 2015 & 2016 & 2019 \\
\hline $\begin{array}{l}\text { Herramienta } \\
\text { de recogida } \\
\text { de datos }\end{array}$ & Bayley-II & Bayley-III & Bayley-III & Bayley-III \\
\hline
\end{tabular}


Necesidades educativas en la etapa infantil en menores con cardiopatías congénitas. Descripción de una cohorte y revisión de la bibliografía

Ma Del Rosario Mendoza Carretero, Susana Ares Segura y Belén Sáenz-Rico De Santiago

\begin{tabular}{|c|c|c|c|c|c|}
\hline & & $\begin{array}{l}\text { Chen et al. } \\
(N=24)\end{array}$ & $\begin{array}{l}\text { Hallioglu et } \\
\text { al. }(N=61)\end{array}$ & $\begin{array}{l}\text { Medoff-Cooper } \\
\text { et al. }(N=72)\end{array}$ & $\begin{array}{l}\text { Este estudio } \\
(\mathrm{N}=90)\end{array}$ \\
\hline \multirow{2}{*}{\multicolumn{2}{|c|}{ 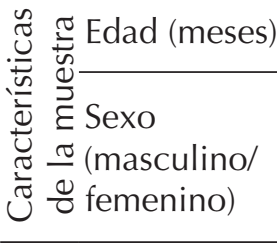 }} & 3 & De 1 a 41 & De 6 a 12 & De 9 a 42 \\
\hline & & $\begin{array}{l}10 \mathrm{CC}(7 / 3) \\
14 \mathrm{GC}(4 / 10)\end{array}$ & $\begin{array}{l}37 \mathrm{CC} \\
(17 / 20) \\
24 \mathrm{GC} \\
(13 / 11) \\
\end{array}$ & $50 / 22$ & $\begin{array}{l}90 \mathrm{CC} \\
61 / 29\end{array}$ \\
\hline \multirow{3}{*}{ 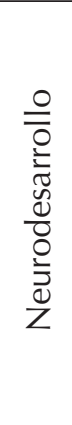 } & Cognitivo & $\begin{array}{l}M=88.50 \pm \\
18.11\end{array}$ & $\begin{array}{l}M=87.36 \\
\pm 16.79\end{array}$ & $\begin{array}{l}6 \mathrm{~m}: \\
M=92 \pm 10 \\
12 \mathrm{~m}: \\
M=94 \pm 12\end{array}$ & $M=99.3$ \\
\hline & Lenguaje & No recogido & $\begin{array}{l}M= \\
87.63 \pm 14.15\end{array}$ & No recogido & $M=87.3$ \\
\hline & Motor & $\begin{array}{l}M=90.10 \pm \\
7.36\end{array}$ & $\begin{array}{l}M= \\
86.66 \pm 17.95\end{array}$ & $\begin{array}{l}6 \mathrm{~m}: \\
M=81 \pm 14 \\
12 \mathrm{~m}: \\
M=80 \pm 16\end{array}$ & $M=91.3$ \\
\hline
\end{tabular}

CC: Cardiopatías congénitas; GC: Grupo Control.

Se presentan cuatro estudios publicados en los últimos cinco años en distintos países: dos en EE.UU, uno en Turquía y otro en España; todos utilizaron como instrumento de evaluación de la Escala de Desarrollo Bayley, segunda y tercera edición. Autores como Gyoung, Young \& Sook (2018) señalan que existe una fuerte correlación entre Bayley-II y BayleyIII en el diagnóstico de infantes con alteraciones en el neurodesarrollo.

El estudio con mayor cohorte $(N=90)$ es el que se realizó en España, valorando a infantes de entre 9 y 42 meses de edad, y el de menor distribución muestral $(N=24)$ es el que se publicó en EE.UU en el 2015, cuya edad de valoración se sitúa en 3 meses de edad.

Los resultados alcanzados por los dos estudios con mayor muestra sitúan el área cognitiva dentro del promedio poblacional, mientras que los que tienen menor población de objeto de estudio, alcanzan puntuaciones inferiores, se sitúan en el rango medio-bajo según la clasificación cualitativa de la propia escala utilizada. El área del lenguaje carece de comparación con dos de los cuatro estudios publicados pues no recogen estos datos; los dos restantes muestran un desarrollo similar $(M=87)$ a pesar de la diferencia muestral $(N=61$ vs. $N=90)$, lo cual puede conducir a una generalización de resultados aunque es necesario ampliar la cohorte de los estudios. No sucede lo mismo con las puntuaciones 
Necesidades educativas en la etapa infantil en menores con cardiopatías congénitas. Descripción de una cohorte y revisión de la bibliografía

Ma Del Rosario Mendoza Carretero, Susana Ares Segura y Belén Sáenz-Rico De Santiago

alcanzadas en el área motora pues son diversas; si bien es cierto, existen similitudes entre el primer y último estudio publicado, así como entre el segundo y el tercero. Sin embargo, las edades de valoración de los cuatro estudios son diferentes, lo cual invita a continuar con la investigación a fin de poder generalizar los resultados encontrados.

Algunos autores como Hallioglu et al., (2016) apoyan que los menores con CC obtienen puntuaciones bajas en todas las escalas. Sin embargo, este estudio muestra que existe un desarrollo normal-lento en el área del lenguaje y que el resto de las mismas se encuentran en un desarrollo normal. A pesar de esto, este estudio carece de comparación con el resto pues los resultados de esta área no están recogidos. De hecho, es difícil encontrar investigaciones que recojan información sobre las tres áreas pues la mayoría se centran en el desarrollo cognitivo y motor (Chen, Harrison, Heathcock, 2015; Medoff-Cooper et al., 2016).

Los participantes de este estudio a nivel cognitivo y motor se encuentran dentro del promedio, aunque no sucede lo mismo con el resto de estudios analizados. Esto sugiere que se continúe investigando para poder generalizar los resultados encontrados. Autores como Calderon et al. (2010), Hövels-Gürich et al. (2007) y Bellinger et al. (2003) evidencian, en edades posteriores, que estos niños presentan dificultades en la memoria de trabajo, en la atención ejecutiva, en la flexibilidad cognitiva, en la resolución abstracta de problemas y en el razonamiento inferencial.

En el área motora, autores como Sananes et al. (2012) destacan que las habilidades motoras finas presentan una disminución significativa según evoluciona el menor y que esto influye negativamente en su desempeño académico. Sin embargo, el desarrollo motor grueso presenta un incremento positivo con el crecimiento del infante. Esto no podemos compararlo con el estudio presentado pues es transversal y no prospectivo. Del resto de estudios, únicamente, el publicado por Medoff-Cooper et al. (2016) muestra el desarrollo del área motora global en dos periodos, a los 6 y a los 12 meses, obteniéndose puntuaciones inferiores en el segundo. Sin embargo, este estudio sí recoge que los menores con CC obtienen mejores puntuaciones escalares en el subárea gruesa.

Estos hallazgos se muestran en las funciones neurocognitivas de orden superior y requieren de estrategias tempranas que permitan limitar estos déficits, siendo necesario realizar un seguimiento, pues éstos se empiezan a mostrar en edades escolares (Creighton et al., 2007). En relación con esto es necesario tener presente el dominio de aprendizaje y 
aplicación de conocimientos publicado en la Clasificación Internacional del Funcionamiento de la Discapacidad y de la Salud, versión para la Infancia y la Adolescencia (CIF-IA) (OMS, 2011). Este dominio describe, entre otras cosas, las experiencias sensoriales intencionadas, como escuchar, mirar, etc.; el aprendizaje básico, como la adquisición del lenguaje, etc., y la aplicación del conocimiento donde adquiere una gran importancia las habilidades atencionales, el pensamiento, la lectura, la escritura, el cálculo, la resolución de problemas y la toma de decisiones, a fin de detectar las limitaciones y restricciones que puedan aparecer.

Se cree que estos déficits pueden deberse a diversos factores biomédicos y ambientales como largos periodos de hospitalización, momento de la intervención quirúrgica (Matsuzaki et al., 2010) y a la duración de la cirugía por circulación extracorpórea (Hövels-Gürich et al., 2007) puesto que se corresponde con un evento traumático que trae consigo cambios en las rutinas de los menores y de sus familias, con una influencia significativa en el desarrollo psicomotor. Sin embargo, este estudio, de los cuatro que se han mostrado en la Tabla 4., es el único que tiene en cuenta la duración de la CEC.

El estudio que se presenta indica que la duración de esta intervención quirúrgica está relacionada con las áreas del desarrollo pues a mayor duración menor desarrollo (véase Figura 2). Independientemente de esta casuística, el área cognitiva se encuentra dentro del promedio poblacional (90-109).

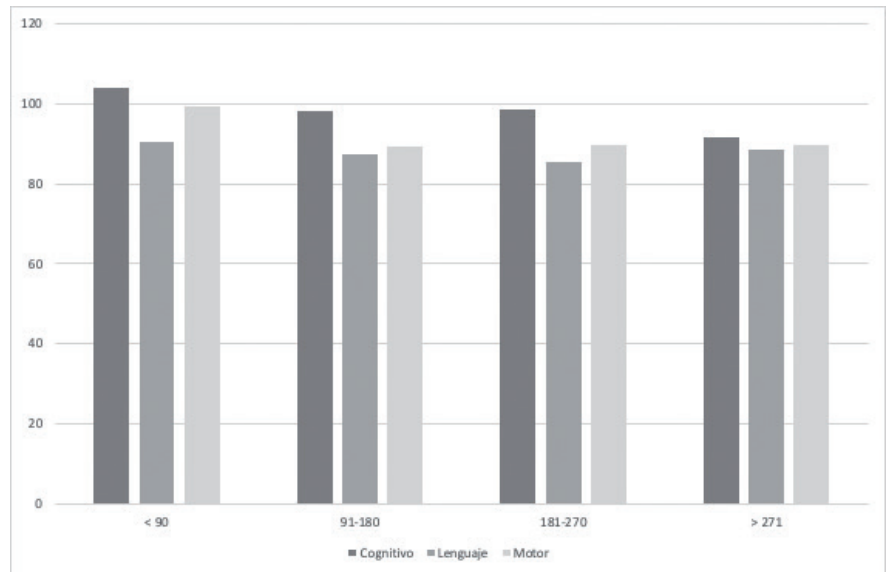

Figura 2. Gráfico de relación entre el tiempo, en minutos, de cirugía por circulación extracorpórea y los resultados alcanzados en el neurodesarrollo. 
Tras el análisis descriptivo reportado con anterioridad, se quiso conocer si existía relación entre las distintas áreas del desarrollo y el tiempo de cirugía con circulación extracorpórea. Los resultados muestran que existe una correlación significativa al nivel .05 entre el tiempo de CEC y el área motora $(p=.03<a=.05)$ (véase Tabla 6). Sin embargo, los factores antropométricos como la talla, el peso, el perímetro cefálico hallados en los estudios consultados muestran que existe riesgo de retraso en el desarrollo neurológico de los menores con CC (Hallioglu et al., 2016; Medoff-Cooper et al., 2016) pero esto no coincide con el estudio presentado.

Tabla 6

Correlaciones entre el tiempo de CEC y las áreas del desarrollo.

\begin{tabular}{|c|c|c|c|c|c|}
\hline & & Cognitivo & Lenguaje & Motor & $\mathrm{T}^{\circ} \mathrm{CEC}$ \\
\hline \multirow{3}{*}{ Cognitivo } & Correlación de Pearson & 1 & $.692^{* *}$ & $.569^{* *}$ & -.181 \\
\hline & Sig. (bilateral) & & .000 & .000 & .107 \\
\hline & $\mathrm{N}$ & 90 & 90 & 90 & 80 \\
\hline \multirow{3}{*}{ Lenguaje } & Correlación de Pearson & $.692^{* *}$ & 1 & $.537^{* *}$ & -.001 \\
\hline & Sig. (bilateral) & .000 & & .000 & .993 \\
\hline & $\mathrm{N}$ & 90 & 90 & 90 & 80 \\
\hline \multirow{3}{*}{ Motor } & Correlación de Pearson & $.569^{* *}$ & $.537^{* *}$ & 1 & $-.238^{*}$ \\
\hline & Sig. (bilateral) & .000 & .000 & & .033 \\
\hline & $\mathrm{N}$ & 90 & 90 & 90 & 80 \\
\hline \multirow{3}{*}{$\mathrm{T}^{\mathrm{o}} \mathrm{CEC}$} & Correlación de Pearson & -.181 & -.001 & $-.238^{*}$ & 1 \\
\hline & Sig. (bilateral) & .107 & .993 & .033 & \\
\hline & $\mathrm{N}$ & 80 & 80 & 80 & 80 \\
\hline
\end{tabular}

**La correlación es significativa en el nivel .01 (bilateral).

*La correlación es significativa en el nivel .05 (bilateral).

Por todo ello, es necesario que estos menores sean incorporados a programas de AT para tratar las necesidades transitorias y permanentes que puedan tener (Sananes et al., 2012; Wernovsky, 2006; MartínezBiarge et al., 2013). A pesar de la evidente necesidad, en España, algunas Comunidades Autónomas como la de Madrid, que cuenta con una de las redes hospitalarias más significativas, no incorpora en los criterios de Ayuda a la Derivación a Recursos de Atención Temprana a aquellos menores que presentan esta enfermedad crónica. Sin embargo, otras comunidades como Galicia (Xunta de Galicia, s.f.) y Andalucía (Pons et al., 
2012), los reconocen como población de riesgo, siendo necesario su participación e inclusión en programas de AT.

\section{Conclusiones}

Gracias a los avances en cardiología pediátrica, a las nuevas técnicas de cirugía cardiovascular y a los cuidados perioperatorios (Hallioglu et al., 2015) se han reducido los índices de mortalidad infantil. Esto ha permitido analizar el neurodesarrollo de los menores con esta patología crónica a través de diversas investigaciones científicas que evidencian la presencia de morbilidades en esta población.

En este caso, es importante mencionar que tanto el área comunicativa-lingüística como la motora, tanto en el estudio que se presenta como en la revisión bibliográfica realizada son las dimensiones del desarrollo que han resultado con mayor significatividad estadística, lo que se traduce en una predisposición para esta población a presentar necesidades en tales áreas. Se observa como la prolongación quirúrgica a partir de 91 minutos afecta a estas áreas del desarrollo, situándose en un nivel medio-bajo (89-80) según la clasificación cualitativa de la Escala de Desarrollo Infantil Bayley-III, mientras que, en las intervenciones que tienen una duración igual o inferior a 90 minutos, los infantes alcanzan mejores resultados en las mencionadas áreas.

Para tratar las necesidades transitorias o permanentes que se puedan generar es preciso realizar un seguimiento de su desarrollo (Rollins y Newburguer, 2014), siendo conveniente realizar una valoración en distintos rangos de edad (12 meses, 24 meses, 36 meses, 6-7 años, 10-11 años), coincidiendo, algunos de ellos, con la incorporación a las distintas etapas educativas como Ed. Infantil (36 meses) y Ed. Primaria (6-7 años). De esta forma se podría detectar la presencia de dificultades de aprendizaje e identificar su área, a fin de dotarles de apoyos educativos que se ajusten a las necesidades que presentan y, que permitan reconducirles positivamente en su desarrollo.

Actualmente, la investigación que se presenta es el resultado de un estudio transversal y sería preciso realizar una investigación de carácter prospectivo, que mostrara el perfil de neurodesarrollo de estos menores a distintos rangos de edad.

Con los resultados alcanzados, se quiere incidir en que desde la ad- 
Necesidades educativas en la etapa infantil en menores con cardiopatías congénitas. Descripción de una cohorte y revisión de la bibliografía

Ma Del Rosario Mendoza Carretero, Susana Ares Segura y Belén Sáenz-Rico De Santiago

ministración estos menores sean derivados a programas de AT tras su nacimiento e intervención quirúrgica en la Comunidad de Madrid, al no ser catalogados en la actualidad como sujetos de riesgo para su inclusión en estos programas dentro de esta Comunidad.

\section{Referencias}

Azcoaga, J.E. (1979). Aprendizaje fisiológico y aprendizaje pedagógico. Buenos Aires: Paidós.

Bellinger, D. C., \& Newburguer, J.W. (2010). Neuropsychological, psychosocial, and quality-of-life outcomes in children and adolescents with congenital heart disease. Prog Pediatr Cardiol, 29(2), 87-92. https://doi.org/10.1016/j.ppedcard.2010.06.007

Bellinger, D.C., Wypij, D., duPlessis, A.J., Rappaport, L.A., Jonas, R.A., Wernovsky, G., \& Newburguer, J.W. (2003). Neurodevelopmental status at eight years in children with dextro-transposition of the great arteries: the Boston Circulatory Arrest Trial. J Thorac Cardiovasc Surg, 126(5), 1385-96. https://doi.org/10.1016/s0022-5223(03)00711-6

Bishop, D. (2006). What causes specific language impairment in children? Current Directions in Psychological Science, 15 (5), 217-221. https://dx.doi. org/10.1111\%2Fj.1467-8721.2006.00439.x

Blair, C., \& Razza, R.P. (2007). Relating effortful control, executive function, and false belief understanding to emerging match and literacy ability in kindegarten. Child Development, 78(2), 647-663. https://doi.org/10.1111/j.1467-8624.2007.01019.x

Calderon, J., Bonnet, D., Courtin, C., Concordet, S., Plumet, M., \& Angeard, N. (2010). Executive function and theory of mind in school-aged children after neonatal corrective cardiac surgery for transposition of the great arteries. Dev Med Child Neurol, 52 (12), 1139-1144. https://doi.org/10.1111/j.1469-8749.2010.03735.x

Calderon, J., Angeard, N., Moutier, S., Plumet, M.H., Jambaqué, I., \& Bonnet, D. (2012). Impact of prenatal diagnosis on neurocognitive outcomes in children with transposition of the great arteries. The Journal of Pediatrics, 161(1), 94-98. https://doi. org/10.1016/j.jpeds.2011.12.036

Cassidy, A.R., White, M.T., DeMaso, D.R., Newburguer, J.W., \& Bellinger, D.C. (2014). Executive function in children and adolescents with critical cyanotic congenital heart disease. J Int Neuropsychol Soc, 21(1), 34-49. https://doi.org/10.1017/ S1355617714001027

Chen, C., Harrison, T., \& Heathcock, J. (2015). Infants with complex congenital heart diseases show por short-term memory in the mobile paradigm at 3 months of age. Infant Behavior and Development, 40, 12-19. http://dx.doi.org/10.1016/j.infbeh.2015.02.007

Comunidad de Madrid. (2018). Protocolo de Coordinación de Atención Temprana. Disponible en: http://www.madrid.org/bvirtual/BVCM014106.pdf

Creighton, D.E., Robertson, C.M., Sauve, R.S., Moddemann, D.M., Alton, G.Y., NettelAguirre, A.,...Western Canadian Complex Pediatric Therapies Follow-up Group. 
Necesidades educativas en la etapa infantil en menores con cardiopatías congénitas. Descripción de una cohorte y revisión de la bibliografía

Ma Del Rosario Mendoza Carretero, Susana Ares Segura y Belén Sáenz-Rico De Santiago

(2007). Neurocognitive, functional and health outcomes at 5 years of age for children after complex cardiac surgery at 6 weeks of age or younger. Pediatrics, 120 (3), e47886. https://doi.org/10.1542/peds.2006-3250

Fundación Salud Infantil. (2019). Cardiopatías congénitas en el recién nacido. Disponible en: https://www.fundacionsaludinfantil.org/cardiopatias-congenitas-en-el-reciennacido/

García, D. (2006). Causas de las cardiopatías. Factores de riesgo. Prevención. En J.Santos. (Ed.), Manual para padres de niños con cardiopatía congénita (pp.70-75). España: lbáñez y Plaza.

Granberg, M., Rydberg, A., \& Fisher, A.G. (2008). Activities in daily living and schooIwork task performance in children with complex congenital heart disease. Acta Paediatr, 97(9):1270-1274. https://doi.org/10.1111/j.1651-2227.2008.00880.x

Greco, R. (2016). Jornada de Cardiopatías Congénitas. Situación actual de la cirugía cardiaca de las principales cardiopatías. Madrid, España. Disponible en: http:// original.livestream.com/grupocto/video?clipld=pla_cded1b26-a689-470b-bdd429f1f9024a3a\&utm_source=Islibrary\&utm_medium=ui-thumb

Gyoung, Y., Young, I. \& Sook, J. (2018). Comparison of second and third editions of the Bayley Scales in Children with suspected developmental delay. Ann Rehabil Med, 42(2): 313-320. https://doi.org/10.5535/arm.2018.42.2.313

Hövels-Gürich, H.H., \& McCusker, C. (2016). Neurodevelopmental Patterns in Congenital heart disease across childhood: Longitudinal Studies from Europe. En C. McCusker y F. Casey. (Ed.), Congenital Heart Disease and Neurodevelopment. Understanding and Improving Outcomes (pp. 41-54). UK: Elsevier. http//dx.doi.org/10.1016/B9780-12-801640-4.00004-4

Hövels-Gürich, H.H., Konrad, K., Skorzenski, D., Minkenberg, R., Herpertz-Dahlmann, B., Messmer, B.J., \& Seghaye, M.C. (2007). Long-term behavior and quality of life after corrective cardiac surgery in infancy for tetralogy of Fallot or ventricular septal defect. Pediatr Cardiol, 28(5), 346-354. https://doi.org/10.1007/s00246-006-0123-z

Hallioglu, O., Gurer, G., Bozlu, G., Karpuz, D., Makharoblidze, K., \& Okuyaz, C. (2015). Evaluation of neurodevelopment using Bayley-III in children with cyanotic or hemodynamically impaired congenital heart disease. Congenit Heart Dis, 10(6), 537-541. https://doi.org/10.1111/chd.12269

Karsdorp, P.A., Everaerd, W., Kindt, M., \& Mulder, B.J. (2007). Psychological and cognitive functioning in children and adolescents with congenital heart disease: a metaanalysis. J Pediatr Psychol, 32(5), 527-541. https://doi.org/10.1093/jpepsy/jsl047

LeRoy, S., Elixson, E.M., O`Brien, P., Tong, E., Turpin, S., Uzark, K.,... Council on Cardiovascular Diseases of the Young. (2003). Recommendations for preparing children and adolescents for invasive cardiac procedures: a statement from the American Heart Association Pediatric Nursing Subcommittee of the Council on Cardiovascular Diseases of the Young. Circulation, 108(20), 2550-2564. https://doi.org/10.1161/01. CIR.0000100561.76609.64

Martín, I. (2006). Supervivencia actual. Secuelas. En J. Santos. (Ed.), Manual para padres de niños con cardiopatía congénita (pp.117-121). España: Ibáñez y Plaza.

Martínez-Biarge, M., Jowett, V.C., Cowan, F.M., \& Wusthoff, C.J. (2013). Neurodevelop- 
Necesidades educativas en la etapa infantil en menores con cardiopatías congénitas.

Descripción de una cohorte y revisión de la bibliografía

Ma Del Rosario Mendoza Carretero, Susana Ares Segura y Belén Sáenz-Rico De Santiago

mental outcome in children with congenital heart disease. Seminars in Fetal \& Neonatal Medicine,18(5), 279-285. https://doi.org/10.1016/j.siny.2013.04.006

Matsuzaki, T., Matsui, M., Ichida, F., Nakazawa, J., Hattori, A., Yoshikosi,K.,...Yagihara, T. (2010). Neurodevelopment in 1-year-old Japanese infants after congenital heart surgery. Pediatr Int, 52(3), 420-427. https://doi.org/10.1111/j.1442200X.2009.02974.x

McCusker, C.G., Armstrong, M.P., Mullen, M., Doherty, N.N., \& Casey, F.A. (2013). A sibling-controlled prospective study of outcomes at home and school in children with severe congenital heart disease. Cardiol Young, 23(4), 507-516. https://doi org/10.1017/S1047951112001667

Medoff-Cooper, B., Iriving, S. Y., Hanlon, A. L., Golfenshtein, N., Radcliffe, J., Stallings, V.A., Marino, B.S., \& Ravishankar, C. (2016). The Association among Feeding Mode, Growth, and Developmental Outcomes in Infants with Complex Congenital Heart Disease at 6 and 12 Months of Age. J Pediatr, 169, 154-159. https://doi.org/10.1016/j. jpeds.2015.10.017

Monfort, M., y Juárez, A. (2010). El niño que habla. El lenguaje oral en preescolar. Madrid: CEPE.

Niakoro. (2019). What is a congenital heart disease? Disponible en: https://www.niakoro. com/families/\#definicion

Organización Mundial de la Salud (OMS). (2020). Anomalías congénitas. Disponible en: https://www.who.int/es/news-room/fact-sheets/detail/congenital-anomalies

Organización Mundial de la Salud (OMS). (2011). Clasificación Internacional del Funcionamiento de la Discapacidad y de la Salud. Versión para la Infancia y la Adolescencia (CIF-IA). Madrid: Ministerio de Sanidad, Política Social e Igualdad. Centro de Publicaciones

Pérez-Lescure, J., Mosquera, M., Latasa, P., y Crespo, D. (2018). Mortalidad de las cardiopatías congénitas en España durante 10 años (2003-2012). Anales de Pediatría, 88(5): 273-279. https://doi.org/10.1016/j.anpedi.2017.06.002

Pons, A., Fornieles, Y., Caballero, R., Campuzano, R., Conejero, J.A., Ferreras, M.J.,... Tapia, M. (2012). Tabla de equivalencies entre la Organización Diagnóstica de Atención Temprana (ODAT) y la Clasificación Internacional de Enfermedades (CIE) [Recurso electrónico]. Sevilla: Consejería de Salud

Real Decreto 46/2015, de 7 de mayo, por el que se regula la coordinación en la prestación de la atención temprana en la Comunidad de Madrid y se establece el procedimiento para determinar la necesidad de atención temprana. Boletín Oficial de la Comunidad de Madrid, 110, 11 de mayo de 2015, pp. 14-24.

Rollins, C.K., \& Newburger, J.W. (2014). Neurodevelopmental outcomes in congenital heart disease. Circulation, 130: e124-e126. https://doi.org/10.1161/CIRCULATIONAHA.114.008556

Salamanca-Zarzuela, B., Morales-Luego, F., Alcalde-Martín, C., y Centeno-Malfaz, F. (2018). Desarrollo psicomotor en pacientes con cardiopatía congénita grave. Revista de Neurología, 66(12): 409-414. https://doi.org/10.33588/rn.6612.2017400

Sananes, R., Manlhiot, C., Kelly, E., Hornberger, L.K., Williams, W.G., MacGregor, D.,... McCrindle, B.W. (2012). Neurodevelopmental outcomes after open heart operations 
Necesidades educativas en la etapa infantil en menores con cardiopatías congénitas. Descripción de una cohorte y revisión de la bibliografía

Ma Del Rosario Mendoza Carretero, Susana Ares Segura y Belén Sáenz-Rico De Santiago

before 3 months of age. Ann Thorac Surg, 93(5), 1577-83. https://doi.org/10.1016/j. athoracsur.2012.02.011

Sociedad Española de Cardiología Pediátrica y Cardiopatías Congénitas (SECPCC). (2014). Manual para padres de niños con cardiopatía congénita. España: Ibáñez\&Plaza

Snookes, S.H., Gunn, J.K., Eldridge, B.J., Donath, S.M., Hunt, R.W., Galea, M.P., \& Shekerdemian, L. (2010). A systematic review of motor and cognitive outcomes after early surgery for congenital heart disease. Pediatrics, 125(4), e818-e827. https://doi. org/10.1542/peds.2009-1959

Villagrá, F. (2019). Cardiopatías congénitas.net: ¿Qué son las cardiopatías congénitas? Madrid, España.: Unidad de Cardiopatías Congénitas. Disponible en: https://cardiopatiascongenitas.net/introcc/quesoncc/

Whitehurst, G. J., \& Fischel, J. E. (1994). Practitioner review: Early developmental language delay: what, if anything, should the clinician do about it? J Child Psychol Psychiatry, 35(4), 613-648. https://doi.org/10.1111/j.1469-7610.1994.tb01210.x

Williams, I.A., Fifer, W.P., \& Andrews, H. (2015). Fetal Growth and Neurodevelopmental Outcome in Congenital Heart Disease. Pediatr Cardiol, 36(6), 1135-1144. doi: 10.1007/s00246-015-1132-6

Xunta de Galicia. (s.f.). Protocolo de coordinación, intervención y derivación interinstitucional en atención temprana. Disponible en: https://escolasaude.sergas.es/Docs/ EGSPC/pilula/Sociosanitarios/resources/protocolo_atencion_temprana.pdf 
